\title{
SYNCHRONIZATION OF A FOUR-WING HYPERCHAOTIC SYSTEM
}

\author{
Masoud Taleb Ziabari ${ }^{1}$ and Ali Reza Sahab ${ }^{2}$ \\ ${ }^{1}$ Faculty of Engineering, Computer Engineering Group, Ahrar University, Rasht, Iran. \\ ${ }^{2}$ Faculty of Engineering, Electrical Engineering Group, Islamic Azad University, Lahijan \\ Branch, Iran.
}

\begin{abstract}
This paper presents synchronization of a four-dimensional autonomous hyperchaotic system based on the generalized augmented Lü system. Based on the Lyapunov stability theory an active control law is derived such that the two four-dimensional autonomous hyper-chaotic systems are to be synchronized. Numerical simulations are presented to demonstrate the effectiveness of the synchronization schemes.
\end{abstract}

\section{KEYWORDS}

Four-dimensional autonomous hyperchaotic system, Lyapunov, Active control, Synchronization.

\section{INTRODUCTION}

Over the last decades, chaos synchronization have been attracted increasingly attentions [1-10]. [2] investigated adaptive control and synchronization of the uncertain Sprott-H system (Sprott, 1994) with unknown parameters. [3] derived new results for the hybrid synchronization of identical hyperchaotic Liu systems (Liu, Liu and Zhang, 2008) via sliding mode control. In hybrid synchronization of master and slave systems, the odd states of the two systems were completely synchronized, while their even states were antisynchronized. the problem of state feedback controller design for the output regulation of Sprott-K chaotic system, which is one of the simple, classical, three-dimensional chaotic systems discovered by J.C. Sprott (1994) was investigated in [4]. [5] presented hyperchaos anti-synchronization of different hyperchaotic systems using Active Generalized Backstepping Method (AGBM). The proposed technique was applied to achieve hyperchaos anti-synchronization for the Lorenz and Lu dynamical systems. In [6], active controller has been designed to solve the output regulation problem for the Sprott-P chaotic system (1994) and a complete solution for the tracking of constant reference signals (setpoint signals). [7] solved the output regulation problem of Sprott-F chaotic system, which is one of the classical chaotic systems discovered by J.C. Sprott (1994). [8] derived new results on the active controller design for the output regulation problem of the Sprott-K chaotic system, which is one of the classical chaotic systems discovered by J.C. Sprott (1994). In [9], chaos synchronization problems of a new 3D chaotic system via three different methods was investigated. Active control and backstepping design methods were adopted when system parameters were known, and adaptive control method was applied when system parameters are unknown. [10] presented chaos synchronization of two different Sprott systems. Chaos synchronization of them by using active control was achieved.

The rest of the paper is organized as follows: In section 2, a four-wing hyperchaotic system is presented. In section 3, synchronization between two four-wing hyperchaotic systems are 
International Journal of Chaos, Control, Modelling and Simulation (IJCCMS) Vol.2, No.3, September 2013

achieved by active control. In section 4, Represents simulation results. Finally, in section 5, Provides conclusion of this work.

\section{SYSTEM DESCRIPTION}

Recently, Xue Wei et al constructed the Four-Wing hyperchaotic system [11]. The system is described by.

$\dot{x}=-\left(\frac{a b}{a+b}\right) x-y z$

$\dot{y}=a y+x z+w$

$\dot{z}=b z+x y+c x+x w$

$\dot{w}=d y$

Where $a, b, c, d$ are positive constants and $x, y, z, w$ are variables of the system, when $a=$ $-35, b=-14, c=1, d=-1$, the system (1) is chaotic. See Figure 1 and Figure 2.

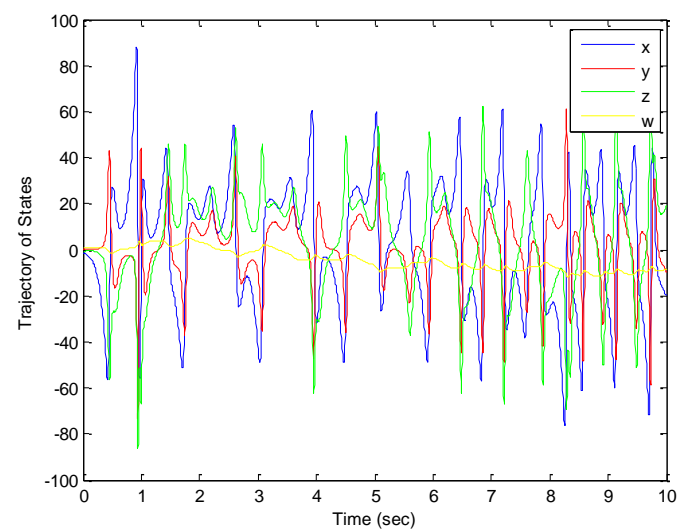

Figure 1. Time response of the system (1).
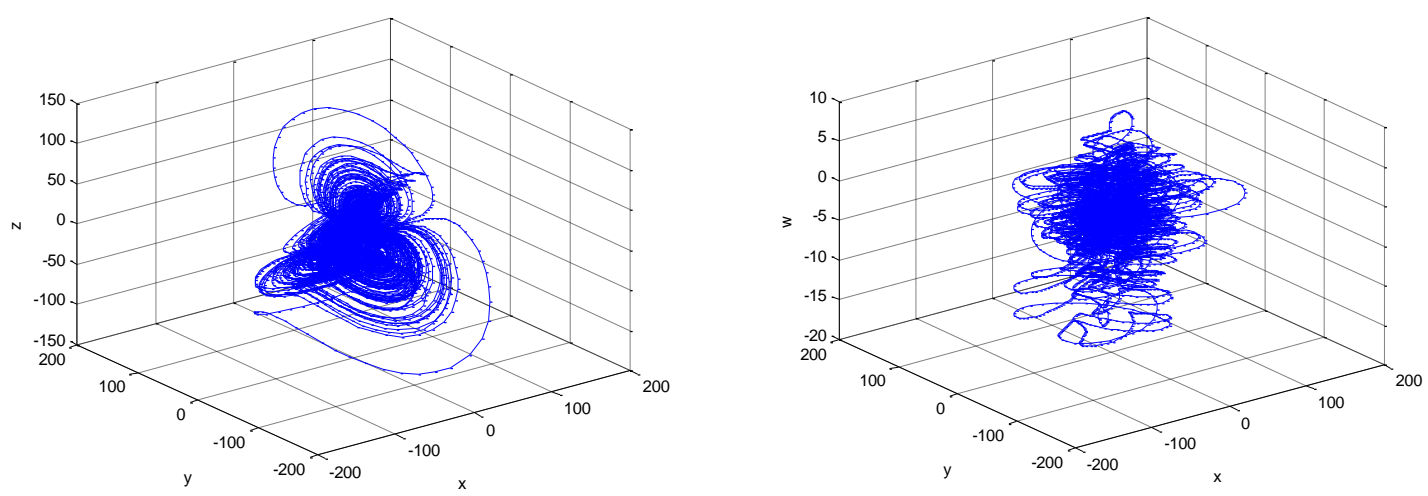

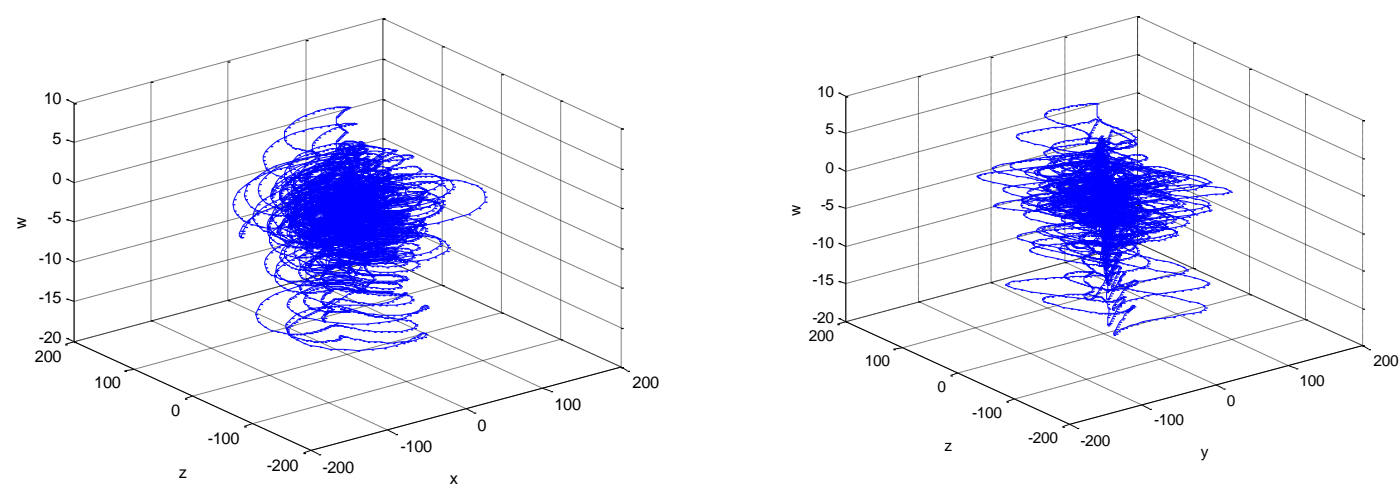

Figure 2. Phase portraits of the four-wing hyperchaotic attractors (1).

\section{SYNCHRONIZATION VIA ACTIVE CONTROL}

In this section, the active control method is applied to synchronize two identical four-wing hyperchaotic systems with known parameters and some global asymptotic synchronization conditions are obtained. Suppose the drive system takes the following form

$\dot{x}_{1}=-\left(\frac{a b}{a+b}\right) x_{1}-y_{1} z_{1}$

$\dot{y}_{1}=a y_{1}+x_{1} z_{1}+w_{1}$

$\dot{z}_{1}=b z_{1}+x_{1} y_{1}+c x_{1}+x_{1} w_{1}$

$\dot{w}_{1}=d y_{1}$

and the response system is given as follows

$\dot{x}_{2}=-\left(\frac{a b}{a+b}\right) x_{2}-y_{2} z_{2}+u_{1}(t)$

$\dot{y}_{2}=a y_{2}+x_{2} z_{2}+w_{2}+u_{2}(t)$

$\dot{z}_{2}=b z_{2}+x_{2} y_{2}+c x_{2}+x_{2} w_{2}+u_{3}(t)$

$\dot{w}_{2}=d y_{2}+u_{4}(t)$

Where $u_{1}(t), u_{2}(t), u_{3}(t), u_{4}(t)$ are control functions to be determined for achieving synchronization between the two systems (2) and (3). Define state errors between systems (2) and (3) as follows

$$
\begin{aligned}
e_{x} & =x_{2}-x_{1} \\
e_{y} & =y_{2}-y_{1} \\
e_{z} & =z_{2}-z_{1} \\
e_{w} & =w_{2}-w_{1}
\end{aligned}
$$

We obtain the following error dynamical system by subtracting the drive system (2) from the response system (3)

$$
\begin{aligned}
& \dot{e}_{x}=-\left(\frac{a b}{a+b}\right) e_{x}-\left(y_{2} z_{2}-y_{1} z_{1}\right)+u_{1}(t) \\
& \dot{e}_{y}=a e_{y}+e_{w}+x_{2} z_{2}-x_{1} z_{1}+u_{2}(t) \\
& \dot{e}_{z}=b e_{z}+c e_{x}+x_{2} y_{2}-x_{1} y_{1}+x_{2} w_{2}-x_{1} w_{1}+u_{3}(t) \\
& \dot{e}_{w}=d e_{y}+u_{4}(t)
\end{aligned}
$$

Define the following active control functions $u_{1}(t), u_{2}(t), u_{3}(t), u_{4}(t)$ 


$$
\begin{aligned}
& u_{1}(t)=y_{2} z_{2}-y_{1} z_{1}+v_{1}(t) \\
& u_{2}(t)=x_{1} z_{1}-x_{2} z_{2}+v_{2}(t) \\
& u_{3}(t)=x_{1} y_{1}-x_{2} y_{2}+x_{1} w_{1}-x_{2} w_{2}+v_{3}(t) \\
& u_{4}(t)=v_{4}(t)
\end{aligned}
$$

Where $v_{1}(t), v_{2}(t), v_{3}(t), v_{4}(t)$ are control inputs. Substituting equation (6) into equation (5) yields

$$
\begin{aligned}
& \dot{e}_{x}=-\left(\frac{a b}{a+b}\right) e_{x}+v_{1}(t) \\
& \dot{e}_{y}=a e_{y}+e_{w}+v_{2}(t) \\
& \dot{e}_{z}=b e_{z}+c e_{x}+v_{3}(t) \\
& \dot{e}_{w}=d e_{y}+v_{4}(t)
\end{aligned}
$$

Thus, the error system (7) to be controlled with control inputs $v_{1}(t), v_{2}(t), v_{3}(t)$ and $v_{4}(t)$ as functions of error states $e_{x}, e_{y}, e_{z}$ and $e_{w}$. When system (7) is stabilized by control inputs $v_{1}(t), v_{2}(t), v_{3}(t)$ and $v_{4}(t), e_{x}, e_{y}, e_{z}$ and $e_{w}$ will converage to zeroes as time $t$ tends to infinity. Which implies that system (2) and (3) are synchronized. To achieve this purpose, we choose control inputs by using active control such that

$v_{1}(t)=\left(\frac{a b}{a+b}-k_{1}\right) e_{x}$

$v_{2}(t)=-\left(a+k_{2}\right) e_{y}-e_{w}$

$v_{3}(t)=\left(b-k_{3}\right) e_{z}-c e_{x}$

$v_{4}(t)=-k_{4} e_{w}-d e_{y}$

we select the gains of controllers (6) in the following form

$k_{1}=10, k_{2}=9, k_{3}=10, k_{4}=7$

And Lyapunov function as

$V\left(e_{x}, e_{y}, e_{z}, e_{w}\right)=\frac{1}{2} e_{x}+\frac{1}{2} e_{y}+\frac{1}{2} e_{z}+\frac{1}{2} e_{w}$

Therefore, the function $\dot{V}\left(e_{x}, e_{y}, e_{z}, e_{w}\right)$ would be obtained negative definite

$\dot{V}\left(e_{x}, e_{y}, e_{z}, e_{w}\right)=-k_{1} e_{x}-k_{2} e_{y}-k_{3} e_{z}-k_{4} e_{w}$

\section{NUMERICAL SIMULATION}

The active control is used as an approach to synchronize two four-wing hyperchaotic systems. The initial values of the drive and response systems are $x_{1}(0)=-9, y_{1}(0)=5, z_{1}(0)=$ $-6, w_{1}(0)=7$ and $x_{2}(0)=8, y_{2}(0)=-7, z_{2}(0)=8, w_{2}(0)=-9$ respectively. The time response of $x, y, z, w$ states for drive system (2) and the response system (3) via active control shown in Figure 3 to Figure 6. Synchronization errors $\left(e_{x}, e_{y}, e_{z}, e_{w}\right)$ in the four-wing hyperchaotic systems shown in Figure 7 . The time response of the control inputs $\left(u_{1}, u_{2}, u_{3}, u_{4}\right)$ for the synchronization four-wing hyperchaotic systems shown in Figure 8. 
International Journal of Chaos, Control, Modelling and Simulation (IJCCMS) Vol.2, No.3, September 2013

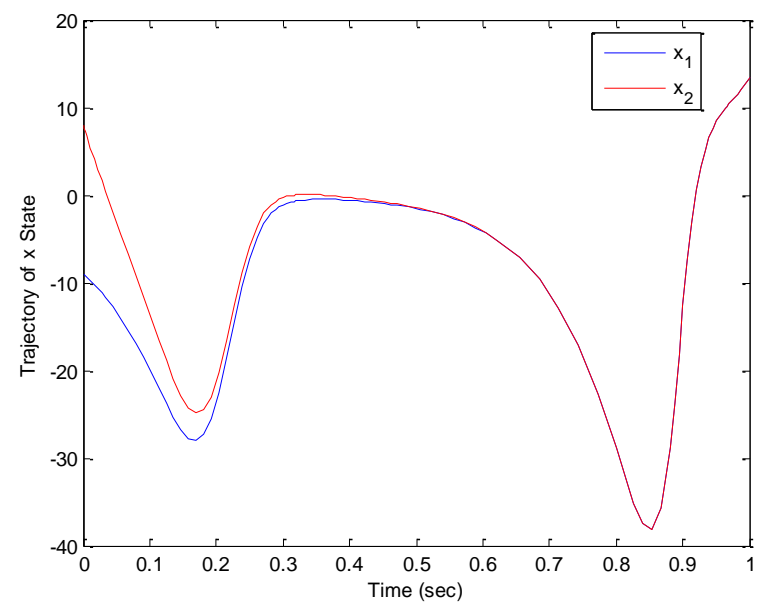

Figure 3. The time response of signal ( $x$ ) for drive system (2) and response system (3).

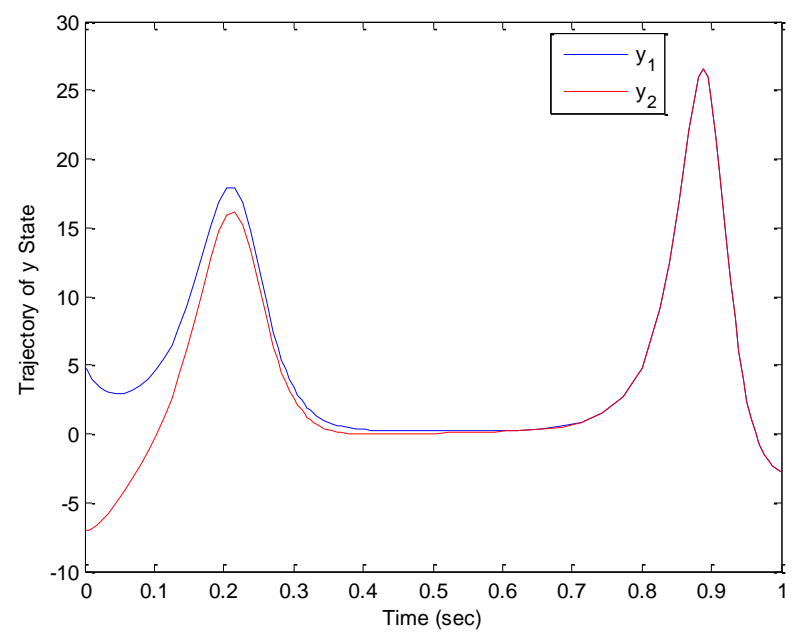

Figure 4. The time response of signal ( $y$ ) for drive system (2) and response system (3).

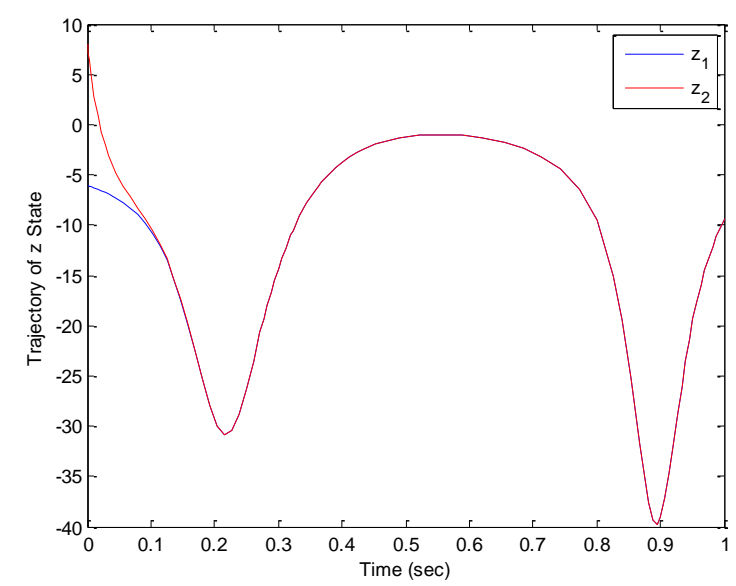

Figure 5. The time response of signal ( $z$ ) for drive system (2) and response system (3). 
International Journal of Chaos, Control, Modelling and Simulation (IJCCMS) Vol.2, No.3, September 2013

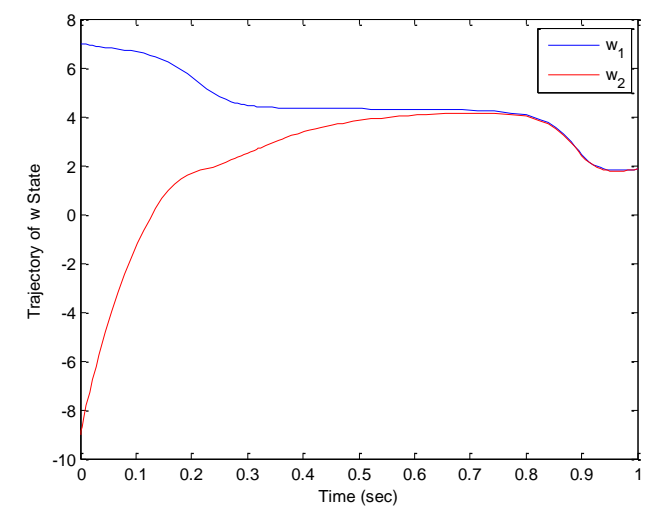

Figure 6. The time response of signal $(w)$ for drive system (2) and response system (3).

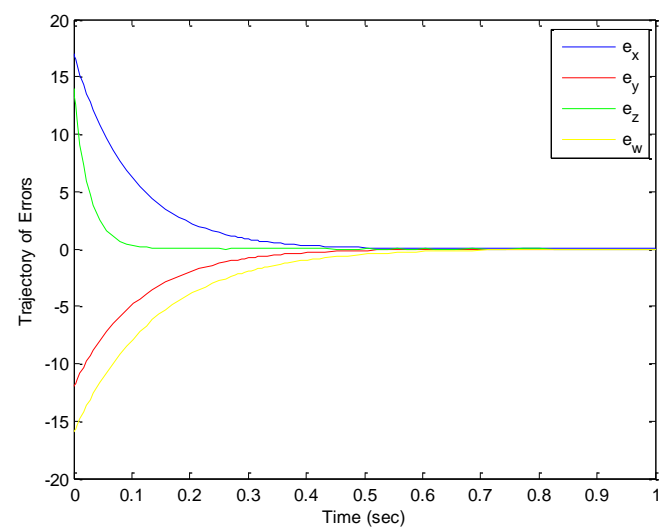

Figure 7. Synchronization errors $\left(e_{x}, e_{y}, e_{z}, e_{w}\right)$ in drive system (2) and response system (3).

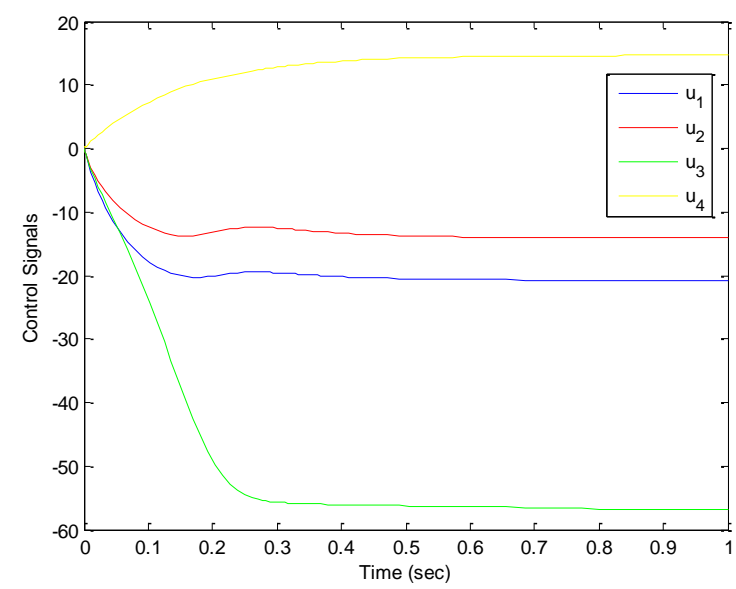

Figure 8. The time response of the control inputs $\left(u_{1}, u_{2}, u_{3}, u_{4}\right)$ for drive system (2) and response system (3).

\section{CONCLUSIONS}

We investigate chaos synchronization of a four-dimensional autonomous hyperchaotic system based on the generalized augmented Lü system via active control in this paper. Based on the Lyapunov stability theory, corresponding controller is designed to achieve synchronization between two identical four-wing hyperchaotic systems. Numerical simulations show that the proposed method work effectively. 


\section{REFERENCES}

[1] Pecora, Carroll. Synchronization in Chaotic Systems. Phys.Rev. Lett,64, (1990) 821-823.

[2] Dr. V. Sundarapandian, Adaptive Control and Synchronization of the Uncertain Sprott H System, International Journal of Advances in Science and Technology, Vol. 2, No.4, 2011.

[3] Sundarapandian Vaidyanathan, HYBRID SYNCHRONIZATION OF HYPERCHAOTIC LIU SYSTEMS VIA SLIDING MODE CONTROL, International Journal of Chaos, Control, Modelling and Simulation (IJCCMS) Vol.1, No.1, September 2012.

[4] Sundarapandian Vaidyanathan, STATE FEEDBACK CONTROLLER DESIGN FOR THE OUTPUT REGULATION OF SPROTT-H SYSTEM, International Journal of Information Sciences and Techniques (IJIST) Vol.1, No.3, November 2011.

[5] Ali Reza Sahab and Masoud Taleb Ziabari, ANTI-SYNCHRONIZATON OF TWO DIFFERENT HYPERCHAOTIC SYSTEMS VIA ACTIVE GENERALIZED BACKSTEPPING METHOD, International Journal of Chaos, Control, Modelling and Simulation (IJCCMS) Vol.2, No.1, March 2013.

[6] Sundarapandian Vaidyanathan, ACTIVE CONTROLLER DESIGN FOR REGULATING THE OUTPUT OF THE SPROTT-P SYSTEM, International Journal of Chaos, Control, Modelling and Simulation (IJCCMS) Vol.2, No.1, March 2013.

[7] Sundarapandian Vaidyanathan, OUTPUT REGULATION OF SPROTT-F CHAOTIC SYSTEM BY STATE FEEDBACK CONTROL, International Journal of Control Theory and Computer Modelling (IJCTCM) Vol.2, No.2, March 2012,.

[8] Sundarapandian Vaidyanathan, ACTIVE CONTROLLER DESIGN FOR THE OUTPUT REGULATION OF SPROTT-K CHAOTIC SYSTEM, Computer Science \& Engineering: An International Journal (CSEIJ), Vol.2, No.3, June 2012.

[9] Yue Wu, Xiaobing Zhou, Jia Chen, Bei Hui, Chaos synchronization of a new 3D chaotic system, Chaos, Solitons and Fractals 42 (2009) 1812-1819.

[10] Dengguo Xu, Chaos Synchronization between Two Different Sprott Systems, Adv. Theor. Appl. Mech., Vol. 3, 2010, no. 4, 195 - 201.

[11] Xue Wei, Fang Yunfei, Li Qiang, A Novel Four-Wing Hyper-Chaotic System and Its Circuit Implementation, 2012 International Workshop on Information and Electronics Engineering (IWIEE), Procedia Engineering 29 (2012) 1264-1269. 